\title{
Design performance requirements and failure analysis of oxygen system on civil aircraft
}

\author{
Jian Wang ${ }^{1 *}$ \\ ${ }^{1}$ Shanghai Aircraft Design and Research Institute, Shanghai, China
}

\begin{abstract}
As an important system for maintaining the respiration and safety of people on board, the oxygen system is widely used in modern civil aircraft. This paper provides a complete overview of the system design performance requirements, starting from the basic design theory of the civil aircraft oxygen system. It also analyzes the failure causes of the oxygen system from the perspective of test flight and operation, and provides some references for the analysis of the failure of the civil aircraft oxygen system and the debugging methods.
\end{abstract}

\section{INTRODUCTION}

Aircraft oxygen systems provide the crew and passengers with vital oxygen in the event of cabin pressurisation failure, thus ensuring the safety of the crew and passengers.

\section{PERFORMANCE REQUIREMENT FOR OXYGEN SYSTEM}

The design of the oxygen system for civil aircraft needs to take into account the main Civil Aviation Administration of China (CAAC) ministerial regulations in force from the date of application for the type, the relevant airworthiness requirements of the US Federal Aviation Administration (FAA) and European Aviation Safety (EASA), as well as any regulations issued by CAAC after the date of application that require retroactive changes. In addition, other relevant new regulations that the airworthiness authorities consider the oxygen system should comply with should also be included.

\subsection{Oxygen Storage Capacity}

The oxygen system shall have the capacity to provide sufficient respiratory oxygen to all crew in the cockpit at cockpit altitudes in excess of $3048 \mathrm{~m}$ (10000 ft). The passenger cabin is capable of supplying respiratory oxygen to all main crew for at least $50 \%$ of the flight endurance in the event of loss of cabin tightness. If the pressurised cockpit loses its airtightness, the pilot should immediately make an emergency descent to a safe altitude where oxygen supply is not required. If fuel conservation is required, the aircraft should be raised again to a more economical cruising altitude, but not above $7600 \mathrm{~m}$.

The $\mathrm{V}_{\text {total }}$ is normally made up of three components: the amount of oxygen required to meet the emergency of descent profile $\left(\mathrm{V}_{\text {descent }}\right)$, the amount of oxygen required for continuous flight after the emergency descent to
$3048 \mathrm{~m}$ (10000ft) ( $\left.\mathrm{V}_{\text {sustaining }}\right)$ and the amount of oxygen required to manoeuvre the aircraft of one-man operation during flight $\left(\mathrm{V}_{\text {single }}\right)$, which can be calculated using the following equation.

Inside:

$$
\begin{gathered}
V_{\text {total }}=N \times\left(V_{\text {descent }}+V_{\text {sustaining }}\right)+V_{\text {single }} \\
V_{\text {descent }}=\sum_{i=1}^{t} Q_{i} \times T_{i} \\
V_{\text {sustaining }}=Q_{10000} \times T_{1} \\
V_{\text {single }}=Q_{800} \times T_{2}
\end{gathered}
$$

In the formula:

Vdescent: Oxygen requirements of a crew member during an emergency descent.

Qi: Oxygen flow through the masks corresponding to the height of each cabin after adjustment by the regulator in $100 \%$ or emergency mode.

Ti: Flight times for each cabin altitude

Vsustaining: Oxygen demand for normal mode oxygen supply for a crew of 1 at a cockpit altitude of $3048 \mathrm{~m}$ $(10,000 \mathrm{ft})$ for a continuous flight time.

Q10000: 3048m (10000ft), mask in normal mode, by the regulator after the adjustment of the oxygen flow.

T1: Time of flight in 3048m (10000ft).

Vsingle: Oxygen demand of a crew member at the controls of an aircraft.

Q8000: 2438m (8000ft), Adjusted oxygen flow in normal mode.

T2: The time during which the aircraft is operated by a single crew member in the cockpit (usually assumed to be 15 minutes).

$\mathrm{N}$ : Number of crew members in the cockpit 


\subsection{Oxygen separation devices}

The oxygen masks of the unit shall meet the requirements specified in TSO-C78, TSO-C89, TSO-C58 (or TSOC139).

\subsection{Inhalation resistance}

The inhalation resistance in the mask should be as specified in table 1 .

Table 1. The inhalation resistance in the mask

\begin{tabular}{|c|c|}
\hline $\begin{array}{c}\text { Continuous flow output } \\
\mathrm{L} / \mathrm{min}\end{array}$ & $\begin{array}{c}\text { Maximum inhalation } \\
\text { resistance in the mask } \\
\mathrm{kPa}\end{array}$ \\
\hline 20 & 0.25 \\
\hline 70 & 0.57 \\
\hline 100 & 0.87 \\
\hline
\end{tabular}

\subsection{Exhalation resistance}

The exhalation resistance in the mask should be as specified in Table 2.

Table 2. The exhalation resistance in the mask

\begin{tabular}{|c|c|c|}
\hline $\begin{array}{c}\text { Continuous flow } \\
\text { output } \mathrm{L} / \mathrm{min}\end{array}$ & \multicolumn{2}{|c|}{$\begin{array}{c}\text { Maximum exhalation resistance in } \\
\text { the mask } \mathrm{kPa}\end{array}$} \\
\cline { 2 - 3 } & $\begin{array}{c}100 \% \text { mode for } \\
\text { pure oxygen }\end{array}$ & $\begin{array}{c}\text { Emergency mode } \\
\text { for pure oxygen }\end{array}$ \\
\hline 20 & 0.25 & 0.75 \\
\hline 70 & 0.50 & 0.95 \\
\hline 100 & 0.75 & - \\
\hline
\end{tabular}

\subsection{Oxygen supply to the oxygen mask}

The oxygen masks of the units are used in conjunction with the regulators and the minimum oxygen requirements at different heights are shown in the curves in Figure 1. The oxygen regulator shall automatically supply pure oxygen at a height of $8000 \mathrm{~m}$ to $10,000 \mathrm{~m}$. The oxygen regulator should be set so that the mode of supplying pure oxygen can be manually selected at any height.

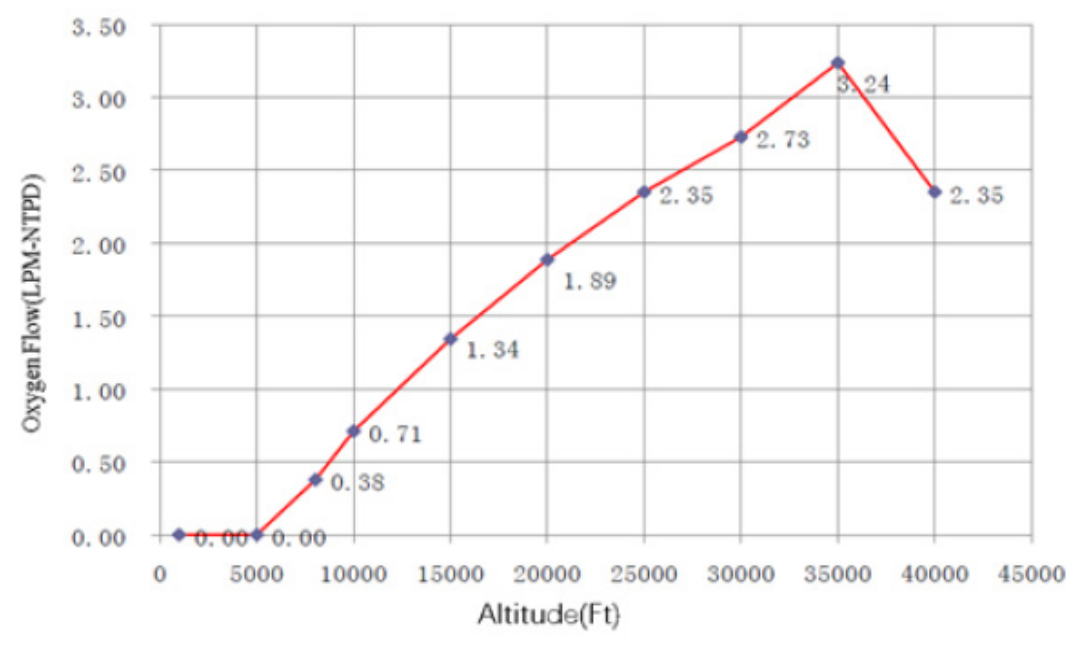

Figure 1. Curve of minimum oxygen supply as a function of height for oxygen masks

\section{ANALYSIS OF OXYGEN'S FAILURE}

\subsection{Analysis of the causes of failure}

The oxygen system is supplied with an oxygen cylinder with a capacity of $2.18 \mathrm{~m} 3(77 \mathrm{ft} 3)$, which supplies oxygen to the full-face oxygen masks via a distribution line. The high-pressure oxygen in the cylinder is converted to lowpressure by the cylinder's own pressure regulator and delivered by a distribution line to the full-face oxygen mask for use by the unit.
The empty oxygen capacity can be judged by the percentage of internal oxygen capacity of the empty oxygen cylinder shown on the summary page of the multifunctional display. The airtightness of the empty oxygen system is such that no more than 5\% leakage is allowed in 24 hours with the empty oxygen cylinder regulator valve open. Failure to do so requires a failure dedue of the air service oxygen system. Factors that commonly affect low oxygen levels include: pilot masks, observer masks, oxygen cylinder components, high and low pressure lines and pipe connections. The fault tree is shown in figure 2. 


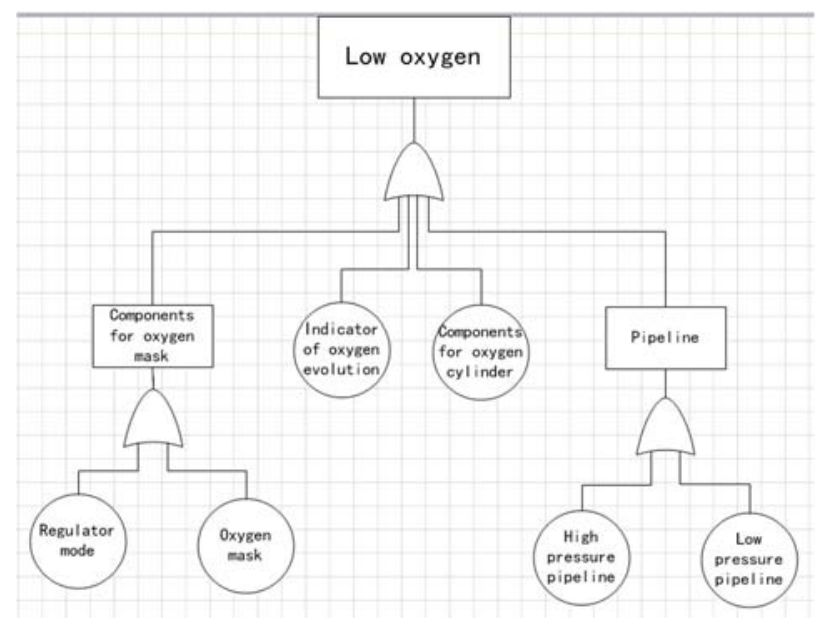

Figure 2. Failure causes of oxygen's leakage

\subsection{Analysis of failure factors}

\subsubsection{Problems with the oxygen evolution's indicator}

The oxygen evolution's indicator outside the machine is a pressure relief device that protects against over pressure. When the pressure inside the cylinder is above $2775 \mathrm{psi}$, the high pressure safety diaphragm on the cylinder assembly ruptures and oxygen is discharged to the outside of the machine through the external discharge line, the external discharge indicator (green disc) is flushed out of the machine, thus providing an indication. If the display shows a zero oxygen level, most of the oxygen discharge indicators outside the machine are faulty or damaged.

\subsubsection{Problems with the regulator mode of oxygen mask}

The oxygen mask regulator has three modes: NORMAL mode, $100 \%$ mode and EMER mode. When the regulator is in NORMAL mode, the gas breathed is a mixture of oxygen and cabin air, and the percentage of oxygen is related to the cockpit pressure altitude. When the regulator is in $100 \%$ mode, the oxygen breathed will always be $100 \%$ oxygen.

In NORMAL and $100 \%$ mode, oxygen only flows during breathing and the flow indicator shows the flow of oxygen with each breath. If the regulator is set to EMER mode, the oxygen will flow continuously and the flow indicator will show continuous flow, resulting in a rapid drop in the oxygen level.

\subsubsection{Problems with oxygen mask}

Oxygen masks supply oxygen to the cockpit crew and comprise the main and co-pilot masks and the observer mask. The oxygen mask consists of the following components: a flexible protective mirror, a regulator adjustment knob, a filling headband, a filling headband switch, a mask oxygen hose, a flow indicator and connector, a mask communication plug, factors affecting leakage from the oxygen mask including the regulator adjustment knob and the filling headband switch.

\subsubsection{Leakage problems in high-pressure pipeline}

Checking high-pressure lines including capillary tubes, Tjunction, pressure gauges, temperature-pressure converters, high-pressure hose assemblies and associated fittings for leaks using nitrogen up to a pressure of 1850 psi. Checking all joints on pressure gauges,T-junction, temperature-pressure converters and capillary tubes for distortion and visible leaks. Stop nitrogen charging to maintain pressure for $1 \mathrm{~h}$ and then check for pressure loss. A pressure loss of more than $5 \mathrm{psi}$ is not sufficient.

\subsubsection{Leakage problems in low pressure pipeline}

Low-pressure pipeline mainly consists of low-pressure pipeline and associated joints, mainly in the EE and cockpit. The piping is complex, with many joints and poor accessibility. At the same time, it places high demands on workers to tighten the pipe joints. There may be leaks despite tightening. When installing the ducting, it should be ensured that the centre line of the duct and the fitting are aligned.

\subsubsection{Component problems with aircraft oxygen cylinders}

The body of an aircraft oxygen cylinder is a composite cylinder made by tightly winding an aluminum inner liner with high-strength epoxy carbon fibre. The components of the aircraft oxygen cylinder that can potentially leak include: the regulator with the ON/OFF knob switch, the pressure gauge, the oxygen filling valve, the oxygen release line connections outside the aircraft, the lowpressure oxygen hose connections and the capillary tube assembly connections. In the event of a high-pressure oxygen leak, the leaked oxygen will be vented directly from the oxygen tank to the outside of the aircraft through the upper vent of the ground-based oxygen service panel. 


\section{Summary}

In this paper, the design performance requirements of the oxygen system of civil aircraft are analysed, and the causes of the functional failure of the oxygen system are also analysed. It is hoped that the procedures and methods proposed in this paper will be of good guidance and reference for civil aircraft test flights and flight line maintenance work.

\section{References}

1. "Aircraft Design Manual" General Editorial Committee. Aircraft Design Manual (Vol. 11). Interior of civil aircraft[M]. Beijing Aviation Industry Press, 2002.

2. Junzhe Yao. A study on the monitoring of crew oxygen system leakage on Airbus A320 aircraft [J]. Aviation Maintenance and Engineering. 2018.79-82

3. Xu Tang. Safety design and assessment of oxygen systems for civil aviation [J]. Scientific and technical information. 2011 (22)

4. Shcheglov. Heterogeneous equilibria in the rheniumoxygen system. 2006 .

5. W.H.Han, M.Miyake, T.Nagai, M.Maeda. Measurements of vapor pressure of $\mathrm{Y}$ and activity of Y-O alloy by multi-Knudsen cell mass.2009 1-2. 